\title{
Analysis of a Case of Brugada Syndrome Through Numerical Simulation of Ventricular Action Potential
}

\author{
Giulia Guidi ${ }^{1}$, Chiara Bartolucci ${ }^{1}$, Anthony Frosio ${ }^{2}$, Procolo Marchese ${ }^{3}$, Annalisa Bucchi ${ }^{2}$, Mirko \\ Baruscotti $^{2}$, Stefano Severi ${ }^{1}$ \\ ${ }^{1}$ University of Bologna, Cesena, Italy \\ ${ }^{2}$ University of Milano, Milano, Italy \\ ${ }^{3}$ Mazzoni Hospital, Ascoli Piceno, Italy
}

\begin{abstract}
Brugada syndrome $(\mathrm{BrS})$ is a disorder characterized by cardiac conduction system dysfunctions, which increases the risk of sudden cardiac death, without heart structural alterations. The diagnosis is based on the ECG tracing analysis: $\mathrm{BrS}$ patients have common anomalies. The typical ECG pattern, however, can remain latent and patients can be asymptomatic: the first symptom often coincides with death.

Recently, a new mutation associated with BrS has been identified and characterized by HEK 293 cells.

This study aims to analyse, through numerical simulation of the O'Hara-Rudy (ORd) model, the mutation effects on the ventricular action potential (AP).

Under normal conditions, the simulation results do not show significant alterations in the mutant AP. For this reason, we hypothesized that the pathological $\mathrm{BrS}$ phenotype could be triggered by other factors.
\end{abstract}

\section{Introduction}

Brugada Syndrome was introduced as a clinical entity in 1992 by the Brugada brothers [1], from whom it takes its name. It is an autosomal dominant cardiac channelopathy characterized by the abnormal electrical activity of the heart, which can lead to sudden cardiac death due to ventricular arrhythmias, even in young and apparently healthy individuals. BrS patients have common electrocardiographic features, such as right bundle branch block and persistent ST-segment elevation in right precordial leads $[1,2]$. It is responsible for $12 \%$ of all cases of sudden cardiac death (SCD) and up to $20 \%$ of SCD in patients with structurally normal hearts [3].

Most patients remain completely asymptomatic and often the first symptom coincides with death. This fact, and the incomplete understanding of underlying cellular mechanisms, are the main problems of the syndrome. The literature indicates that there are several modulating factors that can play a key role in the BrS manifestation, such as bradycardia and hyperactivation of vagal tone, which could explain why arrhythmic events occur mainly during the night [3].

Since 1998, over 350 syndrome-related pathogenic mutations encoding cardiac sodium, potassium and calcium channels have been identified, but mutations of the SCN5A gene, responsible for the loss of sodium channel function, are the most common genotype found in $\mathrm{BrS}$ patients [4].

Recently, a new mutation in the cardiac sodium channels has been identified in an Italian family affected by BrS. This mutation was characterized at the University of Milano, using HEK 293 cells as an experimental model to express both Wild-Type (WT) and mutated model [5].

The results show that the mutation significantly decreases the peak current density by $35 \%$, and positively shifts the half inactivation voltage of the current by 3.6 $\mathrm{mV}$. These and the following observations refer to the heterologous case, with half mutated and half WT channels.

Therefore, two contrasting phenomena emerged from this study: the shift of the inactivation curve indicates that at the same voltage there are more channels available to open, and this fact contrasts the reduction of the observed current density.

For this reason, and since the in vitro study exclusively characterizes the sodium current, we propose to use numerical simulation to quantify the net effect of the phenomena described on the action potential.

\section{Methods}

The ORd human ventricular AP [6] model was chosen to reproduce both WT and mutated action potential because $\mathrm{BrS}$ is thought to originate in the ventricular area, and it is currently the "gold standard" for in silico human ventricular cellular electrophysiology.

In order to reproduce the mutated condition and compare it with the WT one, the parameters that the 
experimental characterization revealed to be affected by the mutation have been modified.

In particular, to mimic the $35 \%$ decrease in the peak current density due to the mutation, the maximum conductance of the fast component of sodium current $\left(\overline{G_{N a, f a s t}}\right)$ was reduced by the same factor.

Following the same reasoning, the maximum conductance of the late component $\left(\overline{G_{N a, l a t e}}\right)$ was scaled.

With regard to time constant of recovery from inactivation and the parameters of channel kinetics, the mutated values were obtained by adding a delta, calculated as the difference between the WT and mutated experimental measurements (Table 1), to the original values of the model.

The ORd model was implemented in Simulink (Mathworks Inc., Natick, MA, USA) with a variable order solver (ode15s). The simulation time was set at 200 seconds, in order to consider results in steady-state conditions.

\begin{tabular}{ll}
\hline Parameter & Delta $(\mathbf{W T}$ vs BrS) \\
\hline $\boldsymbol{V}_{\mathbf{1} / \mathbf{2}}$ activation & $+0.7 \mathrm{mV}$ \\
$\mathrm{K}$ activation & +0.52 \\
$\boldsymbol{V}_{\mathbf{1} / \mathbf{2}}$ inactivation & $+3.6 \mathrm{mV}$ \\
$\mathrm{K}$ inactivation & -0.43 \\
Time constant & $-0.32 \mathrm{~ms}$ \\
\hline
\end{tabular}

Table 1. Values added to ORd parameters to reproduce the mutated condition, calculated from the results of the experimental characterization.

\section{Results}

\subsection{Mutation effect on action potential}

In normal conditions the effect of the mutation on the AP is particularly limited (Figure 1).
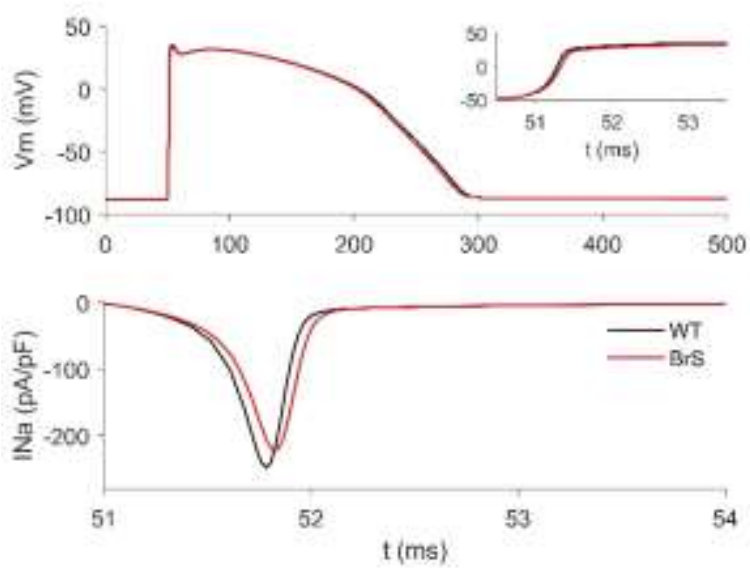

Figure 1. Action potential and sodium current in WT and mutated conditions.
Focusing on the sodium current, the difference between the WT and the mutant peak is about $8 \%$.

From this result the hypothesis which arises is that the loss of function of $I_{\mathrm{Na}}$ may be intensified by other factors. For this reason, we focused our attention to the fact that arrhythmic events due to $\mathrm{BrS}$ are recorded especially in the presence of hyperactivity of the vagal tone, as at night.

\section{2 Acetylcholine effects}

The action of the vagal tone is incisive for several cardiac currents, most of which are specific to cells of the right atrium or the sinoatrial node. One of them is $I_{K A c h}$, a time-independent outward potassium current dependent on Acetylcholine (ACh). It is considered absent in ventricular cells, but a small amount of this current was detected experimentally in the Right Ventricular Outflow Tract (RVOT) of rat cardiomyocytes [8].

The formulation of $I_{K A c h}$ [9] has been added to the ORd model:

$$
\begin{gathered}
\mathrm{I}_{K A c h}=\frac{1}{1+\left(\frac{0,03}{[A c h]}\right)^{2,1}} \cdot\left(0,08+\frac{0,04}{1+\exp \left(\frac{V_{m}+91}{12}\right)}\right) \\
\cdot\left(V_{m}-E_{K}\right)
\end{gathered}
$$

When simulating with an ACh concentration of 10 micromoles, however, there are no effects on the sodium current (Figure 2).
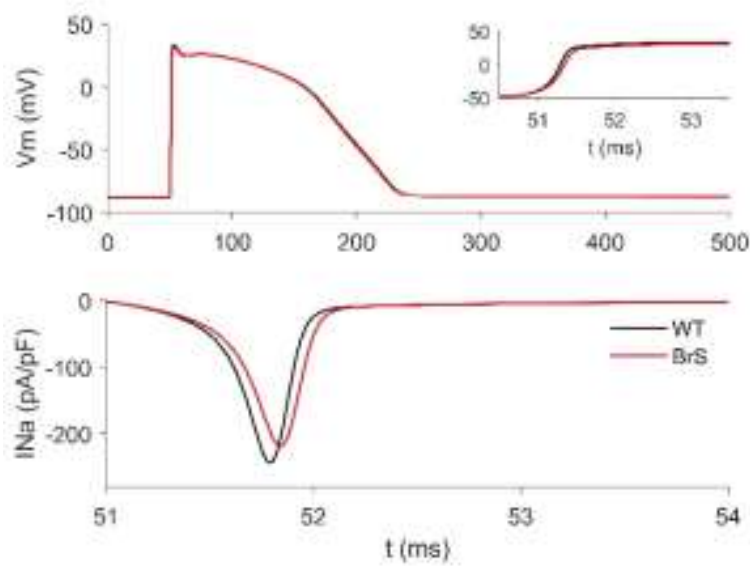

Figure 2. Action potential and sodium current in WT and mutated conditions with $[$ Ach $]=10 \mu \mathrm{M}$.

\section{Discussion and Conclusions}

Numerical simulation has proved useful to investigate the effect of a novel mutation found in BrS patients. In particular, in order to overcome the heterologous system limitations, which does not allow to directly establish the 
impacts of the sodium channel alterations on the cell electrophysiology, we studied the effects of the new mutation associated with the syndrome on ventricular AP.

The results suggest that the two opposite effects of the mutation, i) gain of function due to increased maximal conductance and ii) loss of function due to positive shift of the steady-state inactivation curve, largely compensate each other so that the overall impact on the sodium current under AP is quite limited (-8\% peak current). Such decrease is not likely to provoke arrhythmic episodes.

The occasional occurrence of the Brugada event may depend on other transient factors. In particular, the hypothesis which arises is that the loss of function of $I_{N a}$ might be intensified by the hyperpolarization of the resting potential. This could happen because when moving to more negative potentials, the gap between the WT and mutated inactivation curve decreases. As a consequence, the effect of gain of function decreases, giving way to the loss of function, which might become the dominant effect.

This hypothesis definitely deserves to be further investigated, possibly with a multiscale model, in order to evaluate which arrhythmic consequences are triggered in the ventricle by the possible reduction of sodium current due to the resting hyperpolarization.

\section{References}

[1] Brugada P, Brugada J. Right bundle branch block, persistent ST segment elevation and sudden cardiac death: a distinct clinical and electrocardiographic syndrome. A multicenter report. JACC. 1992;20(6):1391-6.

[2] Brugada R, Campuzano O, Sarquella-Brugada G, Brugada J, Brugada P. Brugada Syndrome. Methodist DeBakey Cardiovascular Journal. 2014;10(1):25-28.

[3] Juang JM, Huang SK. Brugada syndrome-an underrecognized electrical disease in patients with sudden cardiac death. Cardiology. 2004;101(4):157-69.

[4] Berne P, Brugada J. Brugada Syndrome 2012. Circulation Journal. 2012;76:1563-1571.

[5] Frosio A. Functional characterization of new SCN5A mutations associated with different patterns of arrhytmia. PhD thesis at University of Milano-Bicocca.

[6] O'Hara T, Virág L, Varró A, Rudy Y. Simulation of the undiseased human cardiac ventricular action potential: model formulation and experimental validation. PLoS Comput Biol 2011;7.

[7] Attwell D, Cohen I, Eisner DA. The effects of heart rate on the action potential of guinea-pig and human ventricular muscle. J. Physiol. 1981;313: 439-461.

[8] McMorn SO, Harrison SM, Zang WJ, Yu XJ, Boyett MR. A direct negative inotropic effect of acetylcholine on rat ventricular myocytes. The American Physiological Society. 1993;265: H1393-H1400.

[9] Grandi E, Pandit SV, Voigt N, Workman AJ, Dobrev D, Jalife, Bers DM. Human Atrial Action Potential and Ca2+ Model: Sinus Rhythm and Chronic Atrial
Fibrillation. Circulation Research. 2012;109(9):105566.

[10] Gumz ML, Rabinowitz L. Role of Circadian Rhythms in Potassium Homeostasis. Seminars in Nephrology. 2013;33(3): 229-236.

[11] Schmidt ST, Ditting T, Deutsch B, Schutte R, Friedrich $\mathrm{S}$, Kistner I, Ott C, et al. Circadian rhythm and day to day variability of serum potassium concentration: a pilot study. Journal of Nephrology. 2015;28: 165-172.

Address for correspondence:

Stefano Severi

Department of Electrical, Electronic

and Information Engineering,

University of Bologna,

Via dell'Università 50, 47522 Cesena (FC),

Italy

stefano.severi@unibo.it 